\title{
Community-Based Aerobic Exercise Program for Primary Prevention of Cardiovascular Disease in Adults With Visual or Auditory Impairments: A Feasibility Study
} Sora Baek, MD, $\mathrm{PhD}^{1,2,3}$, Yuncheol Ha, PT, $\mathrm{MS}^{1,2}$, Jaemin Mok, $\mathrm{SW}^{1,4}$,
Haekyung Lee, $\mathrm{MPH}^{5}$, Woojoo Song, $\mathrm{PT}^{5}$

\footnotetext{
${ }^{1}$ Kangwon Regional Cardiocerebrovascular Center, Kangwon National University Hospital, Chuncheon; ${ }^{2}$ Department of Rehabilitation Medicine, Kangwon National University Hospital, Chuncheon; ${ }^{3}$ Department of Rehabilitation Medicine, Kangwon National University School of Medicine, Chuncheon; ${ }^{4}$ Public Health and Social Work Office, Kangwon National University Hospital, Chuncheon; ${ }^{5}$ Cheorwon Public Health Center, Cheorwon, Korea
}

Objective To investigate the feasibility of a public health center-based aerobic and resistance training program for primary prevention of cardiovascular disease in people with visual, auditory, or physical/brain impairments.

Methods The study included 25 adults aged $>40$ years who lived in Cheorwon-gun in South Korea, had a disability registered for visual, auditory, or physical/brain impairments under the Disability Welfare Act, and had either known cardiovascular disease or two or more risk factors for cardiovascular disease. The program comprised four education sessions and 12 weeks of customized aerobic and strengthening exercises performed twice a week at moderate intensity, with each exercise session lasting for 1 hour. The body mass index (BMI), percent body fat, 6-minute walk distance (6MWD), and 30-second sit-to-stand test results were measured at baseline and on program completion.

Results Seventeen subjects (68\%) completed the program. There were significant decreases in BMI and percent body fat (both $\mathrm{p}<0.05)$, with a significant increase in 30 -second sit-to-stand strength $(\mathrm{p}<0.05)$ but no changes in the 6MWD. In subjects with visual or auditory impairments, BMI and percent body fat were significantly decreased after the program; however, there was no significant change in the results of the 30-second sit-to-stand strength test or the 6MWD.

Conclusion In people with disabilities, a 3-month community-based exercise program can decrease body mass index and percent body fat and increase sit-to-stand strength. The 30-second sit-to-stand test may be a useful measure of the strength and endurance of the lower extremities in people with disabilities.

Keywords Cardiac rehabilitation, Cardiovascular diseases, Community health centers, Disabled persons, Exercise

Received September 22, 2020; Revised December 18, 2020; Accepted January 28, 2021; Published online June 14, 2021

Corresponding author: Sora Baek

Department of Rehabilitation Medicine, Kangwon National University Hospital, Kangwon National University School of Medicine, 156 Baengnyeong ro, Chuncheon 24289, Korea. Tel: +82-33-258-9262, Fax: +82-33-25-9097, E-mail: sora.baek@kangwon.ac.kr

ORCID: Sora Baek (https://orcid.org/0000-0003-3404-6202); Yuncheol Ha (https://orcid.org/0000-0001-9140-5713); Jaemin Mok (https://orcid. org/0000-0001-6196-7280); Haekyung Lee (https://orcid.org/0000-0002-0818-9157); Woojoo Song (https://orcid.org/0000-0002-6822-580X).

(c) This is an open-access article distributed under the terms of the Creative Commons Attribution Non-Commercial License (http://creativecommons.org/ licenses/by-nc/4.0) which permits unrestricted noncommercial use, distribution, and reproduction in any medium, provided the original work is properly cited. Copyright (c) 2021 by Korean Academy of Rehabilitation Medicine 


\section{INTRODUCTION}

The World Health Organization defines a disability as an impairment of the body or mind (e.g., paralysis or blindness) that limits activity (e.g., seeing, hearing, or walking) and restricts participation (e.g., employment, social and recreational activities, and access to healthcare and preventive services) [1]. There are many types of disability and a range of impairments that involve vision and hearing, mobility, thinking, memory, learning, communication, mental health, and social relationships [1].

A physical disability is a condition that affects a person's mobility, physical capacity, stamina, or dexterity. Examples include brain or spinal cord injury, multiple sclerosis, cerebral palsy, a respiratory disorder, epilepsy, and hearing or visual impairment [2]. People with disabilities are at greater risk of preventable health problems. Children and adults with disabilities are more likely to be obese than able-bodied children and adults. Overweight and obesity can have serious health consequences [3].

There are several known risk factors for cardiovascular events, including hypertension, obesity, dyslipidemia, diabetes, smoking, and a sedentary lifestyle [4]. Moreover, physical disability is associated with increased cardiovascular risk [5]. The coronary event rate is higher in older adults with moderate to severe disability than in those without disability [6]. In a retrospective cohort study of people with disabilities, the risk of acute myocardial infarction was 2.04 times higher in subjects with a severe disability than in those with a mild disability [7]. Furthermore, this risk is reportedly higher in people with a physical disability than in their counterparts with a mental disability [7].

A significant reduction in cardiovascular mortality was demonstrated in patients who underwent cardiac rehabilitation than in controls who did not [8]. Furthermore, cardiac rehabilitation was effective even for people with limited ability to exercise. Resistance training in older patients with coronary heart disease and a reduced physical function score has been shown to improve physical performance [9]. Nevertheless, the frequency with which people with disability use preventive health services is less than ideal [10]. Disability also acts as a barrier to participation in a cardiac rehabilitation program. A study in South Korea showed that comorbidity/functional impair- ment was a significant barrier to cardiac rehabilitation [11].

A community-based exercise program may be useful for primary or secondary prevention of cardiovascular disease in patients who find it difficult to participate in such programs due to comorbidity. The Disability Welfare Act in South Korea recognizes 15 categories of physical or mental disorders [12], which are grouped as follows: external physical disability (physical, brain, visual, auditory, and language impairment and facial deformity), internal organ disability (renal, heart, liver, respiratory, intestinal, and urinary tract dysfunction), and intellectual disability (epilepsy, intellectual disability, developmental delay, and mental impairment) [12,13]. In order for an individual to be registered as having disability, there must be both a diagnosis of the impairment and a decline in function documented in the medical records. Physical impairment includes amputation, joint stiffness or contracture, paralysis due to a cause other than a brain lesion, and deformity. Paralysis secondary to a brain lesion is classified as brain impairment. According to the Korean health and welfare statistical yearbook 2019 [14], 92.7\% of disabled persons in South Korea had a physical impairment (47.9\%), auditory impairment (13.2\%), brain impairment (9.8\%), visual impairment (9.8\%), an intellectual disability (8.0\%), or kidney dysfunction (3.4\%).

People with physical disabilities often encounter transport problems [13] that make it difficult for them to attend for cardiac rehabilitation at a distant hospital. In a previous study, we investigated the feasibility, effectiveness, and safety of community-based cardiac rehabilitation at a public health center for communitydwelling older adults [15]. In this study, we investigated the feasibility of a public health center-based aerobic and resistance exercise program for primary prevention of cardiovascular disease in people with disabilities in Cheorwon-gun, where there is no cardiac rehabilitation center. We hypothesized that people with visual, auditory, or physical/brain impairments would be capable of increasing their muscle strength and aerobic capacity and decreasing their cardiovascular risk if they participated in a community-based exercise program. 


\section{MATERIALS AND METHODS}

\section{Participants}

Potential study subjects were people with disabilities who were allowed to perform moderate-intensity exercise without requiring a pre-participation medical screening test as recommended in the American College of Sports Medicine (ACSM) guideline (Table 1) $[4,16]$. The study inclusion criteria were as follows: community-dwelling; age $>40$ years; a disability registered under the Disability Welfare Act; residence in Cheorwon-gun, Gangwon Province; established cardiovascular disease (secondary prevention) with current regular exercise or the presence of two or more risk factors for cardiovascular disease (primary prevention); and ability to walk independently. Independent walking ability was assessed by the Functional Ambulation Categories (FAC) tool. The FAC is a visual measurement of walking that distinguishes six levels (0-5) of walking ability on the basis of the amount of physical support required and can be performed rapidly [17]. Only subjects capable of independent ambulation (FAC 4-5) were included in this study.

The following exclusion criteria were applied: pain or discomfort in the chest, neck, jaw, arm, or other area that might be caused by ischemia; shortness of breath at rest or on mild exertion; dizziness or syncope; orthopnea or paroxysmal nocturnal dyspnea; ankle edema; palpitations or tachycardia; a known heart murmur; or unusual fatigue or shortness of breath when performing usual activities [4]. Subjects with a score of $<20$ on the Korean version of the Mini-Mental State Examination were also excluded.

Sign language interpreters participated in the exercise program, education program, and evaluation process

Table 1. Criteria for enrollment and discontinuation of exercises and assessments

\begin{tabular}{|c|c|}
\hline & Description \\
\hline \multirow[t]{3}{*}{ Enrollment criteria } & $\begin{array}{l}\text { Community-dwelling adults (aged }>40 \text { years) with disabilities registered under the Dis- } \\
\text { ability Welfare Act }\end{array}$ \\
\hline & $\begin{array}{l}\text { Known cardiovascular/renal/metabolic disease or with two or more risk factors: (1) } \\
\text { known cardiovascular (cardiac, peripheral vascular, or cerebrovascular disease), renal, } \\
\text { or metabolic disease (type } 1 \text { or } 2 \text { diabetes mellitus) who already performed regular exer- } \\
\text { cise }^{\text {a) }} \text { or (2) no known cardiovascular, renal, or metabolic disease but with two or more } \\
\text { risk factors }\end{array}$ \\
\hline & Functional Ambulation Categories 4-5 \\
\hline \multirow[t]{2}{*}{ Exclusion criteria } & Signs or symptoms suggestive of cardiac, peripheral vascular, or cerebrovascular disease \\
\hline & Mini-Mental State Examination score $<20$ \\
\hline \multirow{3}{*}{$\begin{array}{l}\text { Exercise/evaluation } \\
\text { not-participating criteria }\end{array}$} & $\mathrm{BP} \geq 160 / 100 \mathrm{mmHg}$ \\
\hline & Chest pain (angina scale $\geq 1$ ) \\
\hline & Respiratory difficulty (dyspnea scale $\geq 1$ ) \\
\hline \multirow{2}{*}{$\begin{array}{l}\text { Exercise/evaluation } \\
\text { stopping criteria }\end{array}$} & $\mathrm{BP} \geq 250 / 115 \mathrm{mmHg}$ or drop in SBP of $\geq 10 \mathrm{mmHg}$ with an increase in exercise intensity \\
\hline & Chest pain and respiratory difficulty: angina scale $\geq 2$, dyspnea scale $\geq 3$ \\
\hline
\end{tabular}

BP, blood pressure; SBP, systolic blood pressure.

${ }^{a)}$ Regular exercisers should have a history of performing planned and structured physical activity of at least moderate intensity for at least 30 minutes for 3 or more days/week during the previous 3 months [4].

${ }^{b)}$ Risk factors included age ( $\geq 45$ years for men and $\geq 55$ years for women), family history (myocardial infarction, coronary revascularization, or sudden death before 55 years of age in the father or other male first-degree relative or before 65 years of age in the mother or other female first-degree relative), cigarette smoking (current cigarette smoker, those who quit within the previous 6 months, or those exposed to environmental tobacco smoke), sedentary lifestyle (nonparticipation in at least 30 minutes of moderate-intensity physical activity [ $40 \%$ to $<60 \% \mathrm{VO}_{2} \mathrm{R}$ ] for at least 3 days/week for at least 3 months), obesity (body mass index $\geq 25 \mathrm{~kg} / \mathrm{m}^{2}$ or waist circumference $>90 \mathrm{~cm}$ for men and $>85 \mathrm{~cm}$ for women), hypertension, dyslipidemia (total cholesterol $\geq 200 \mathrm{mg} / \mathrm{dL}$, or use of lipid-lowering medications), and prediabetes (glycated hemoglobin of 5.7\%-6.4\%) [16]. 
for subjects with auditory impairment. The study was conducted between May 2019 and September 2019 in accordance with the principles embodied in the Declaration of Helsinki. The study protocol was approved by the Institutional Review Board of Kangwon National University Hospital (No. 2019-03-002-001). Written informed consent was obtained from all study participants.

\section{Community-based aerobic and resistive exercise program}

A community-based aerobic and resistive exercise program was carried out at the public health center in Cheorwon-gun, Gangwon Province. The program was a modified version of a cardiac rehabilitation program previously used at a public health center [15]. It included patient assessment, 12 weeks of customized exercise, and four education sessions (Table 2) according to the core components of cardiac rehabilitation recommended by the American Heart Association and the American Association of Cardiovascular and Pulmonary Rehabilitation [18]. The exercises were performed twice a week at moderate intensity, with each exercise session lasting for 1 hour and comprising 30 minutes of aerobic exercise and 30 minutes of strengthening training under the supervision of physical therapists.

The education program was composed of four sessions.
Session 1 (Understanding heart disease and controlling risk factors) covered myocardial infarction, the benefit of physical activity/exercise, metabolic syndrome, assessment of individual cardiovascular risk factors, and risk factor management. Session 2 (Exercises for prevention and treatment of heart disease) included components of cardiac rehabilitation, indications for cardiac rehabilitation, measurements of heart rate, exercise intensity using heart rate, rating of perceived exertion, rating of chest pain and respiratory difficulty, exercise stages (warm up/ workout/cool down), estimating maximum age-related heart rate, calculating heart rate reserve of $40 \%-60 \%$, and components of exercise prescription (frequency, intensity, time, and duration). In Session 3, the effects of cardiac rehabilitation on mortality, quality of life, risk factor reduction, and cardiopulmonary fitness were discussed. Session 4 addressed exercise precautions needed in people with disabilities.

Aerobic exercise consisted of cycling on a stationary bicycle equipped with a heart rate sensor (Echobike V-501; EunSung Healthvill Co. Ltd., Chilgok-gun, Korea). The subject holds the heart rate sensor on the handle while cycling, and the heart rate is displayed on an LCD monitor. The recommended rating for the perceived exertion score was no more than 11-14 or a heart rate reserve of $40 \%-60 \%$. For the calculation of heart rate reserve, the

Table 2. Education and exercise program

\begin{tabular}{|c|c|c|}
\hline & Education program & Exercise program \\
\hline \multicolumn{3}{|c|}{ Baseline assessment } \\
\hline Week 1 & $\begin{array}{l}\text { Understanding heart disease and } \\
\text { controlling risk factors }\end{array}$ & \multirow{3}{*}{$\begin{array}{l}\text { Frequency: twice a week } \\
\text { Exercise composition: } 30 \text {-minute aerobic exercise/ } \\
\text { 30-minute strengthening exercise }\end{array}$} \\
\hline Week 2 & - & \\
\hline Week 3 & $\begin{array}{l}\text { Exercises for prevention and treatment of } \\
\text { heart disease }\end{array}$ & \\
\hline Week 4 & - & \multirow{3}{*}{$\begin{array}{l}\text { Aerobic exercise } \\
\text { Intensity: RPE } 11-14 \text { (moderate intensity) or HRR } 40 \%-60 \% \\
\text { Type: stationary bicycle }\end{array}$} \\
\hline Week 5 & Effects of cardiac rehabilitation & \\
\hline Week 6 & - & \\
\hline Week 7 & Exercise for people with disabilities & \multirow{6}{*}{$\begin{array}{l}\text { Strengthening exercise } \\
\text { Intensity: RPE 11-14 (moderate intensity) } \\
\text { Type: elastic band (yellow and red color) }\end{array}$} \\
\hline Week 8 & 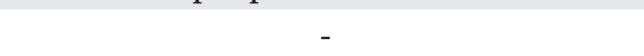 & \\
\hline Week 9 & - & \\
\hline Week 10 & - & \\
\hline Week 11 & - & \\
\hline Week 12 & - & \\
\hline \multicolumn{3}{|c|}{ Final assessment } \\
\hline
\end{tabular}

HRR, heart rate reserve; RPE, rate of perceived exertion. 
age-predicted maximal heart rate derived from the equation (HRmax $=220$ - age) was used [19].

TheraBand (Performance Health, Akron, OH, USA) tubing was used to perform the lower limb, trunk, and upper limb strengthening exercises. The color of the tubing was selected for moderate intensity exercise (perceived exertion score 11-14), ranging from yellow (low resistance) to red (moderate resistance). Subjects could perform TheraBand exercises regardless of type of impairment. The exercises used to strengthen the lower limbs included ankle dorsiflexion, ankle plantar flexion, squat, and hip abduction. Side bending and modified hip extension in the bridge position were performed to strength the trunk muscles. The strengthening exercises used for the upper limb muscles were reverse flies, scapular retraction, and lateral raises [20]. Subjects with auditory impairment performed front squats for the lower limbs, quadruped arm and leg stabilization for the trunk muscles, and bilateral biceps curls for the upper limbs using tubing with loops (TheraBand CLX) [21].

Blood pressure, chest pain, and respiratory difficulty were evaluated before and during each exercise and assessment session. Chest pain and respiratory difficulty were assessed using the angina scale $(0=$ none, $1=$ little, $2=$ medium, $3=$ very, $4=$ extreme) and dyspnea scale ( 0 =none, $1=$ little, $2=$ medium, $3=$ =very, $4=$ =xtreme), respectively. Subjects with high blood pressure at rest and/ or chest pain and respiratory difficulty were not allowed to participate in the exercise or assessment sessions. Exercise or assessment was stopped if blood pressure was $\geq 250 / 115 \mathrm{mmHg}$, the angina scale score was $\geq 2$, the dyspnea scale score was 3 , or upon request by the subject (Table 1).

\section{Outcome measurements}

Outcomes were measured at baseline and after completion of the 12-week program (Table 2). Outcome measurements included functional status, physical activity, quality of life, anthropometric measurements, bioimpedance analysis, resting blood pressure, resting heart rate, blood tests, physical strength, and exercise capacity. Functional status was assessed using the Korean Activity Scale/Index score (range, 0-79) [22]. Physical activity over 7 days was assessed using the Korean version of the International Physical Activity Questionnaire Short Form and calculated as metabolic equivalent of task (MET) minutes per week [23]. Quality of life was measured using the European Quality of Life-5 Dimension-3-Level version (EQ-5D-3L); the EQ-5D-3L index was calculated (range, -0.171-1), with higher values indicating a better health status [24]. Waist circumference $(\mathrm{cm})$, height $(\mathrm{cm})$, and body weight $(\mathrm{kg})$ were measured, and BMI was calculated as weight $(\mathrm{kg})$ divided by the square of the height $\left(\mathrm{m}^{2}\right)$. Fasting blood sugar $(\mathrm{mg} / \mathrm{dL})$, glycated hemoglobin (HbAlc, \%), total cholesterol (mg/dL), and triglycerides $(\mathrm{mg} / \mathrm{dL})$ were measured. Percent body fat $(\%)$ and muscle mass $(\mathrm{kg})$ were measured by bioimpedance analysis using the InBody 770 body composition analyzer (InBody Co. Ltd., Seoul, Korea).

Physical strength was measured by handgrip strength and performance on the 30-second sit-to-stand test. Handgrip strength was assessed using a hydraulic hand dynamometer (SH5001; Saehand Corp., Masan, Korea). Participants was seated comfortably in a chair without arm-rests, and the shoulder was adducted and neutrally rotated with the elbow held at $90^{\circ}$ of flexion and the forearm and wrist maintained in a neutral position. Lower limb strength was measured using the 30-second sit-tostand test, which counts how many times the subject can rise and sit in the chair over a 30-second interval [25]. The subject was seated in a chair without an arm-rest, and then repeated full stand-up and seating for 30 seconds. The participants were encouraged to complete as many full stands as possible within the 30 -second limit, and the total number of stands was counted. A greater number of stands indicates greater quadriceps strength.

Exercise capacity was evaluated using the 6-minute walk distance (6MWD) test following the recommended guidelines [26]. The 6MWD test was supervised by physiotherapists in accordance with the protocol outlined by the American Thoracic Society. The guideline recommends testing in a long corridor; however, considering our study participants' potentially poor balance when walking and their risk of falls, we performed the 6MWD test at an adjacent indoor Judo-gym in which the floor is covered with a cushioned mat rather than a hard floor.

\section{Statistical analysis}

If the subjects attended more than $70 \%$ of the exercise sessions, they were considered to have completed the program. The ages of subjects who did and did not complete the community-based exercise program were 
compared using the Mann-Whitney U test. Sex and type of disability were also compared according to whether subjects did or did not complete the program using the $\chi^{2}$ test.

Subjects who completed the final assessment were included in the outcome analysis. Measurements obtained at baseline and after the 12-week exercise program were compared using the paired-t test. Considering the wide range of disabilities in our study participants, we compared the outcome measurements at baseline and upon completion by subgroup according to whether the impairment was visual or auditory using the Wilcoxon signed-rank test. The statistical analysis was performed using jamovi version 1.1.9 (https://www.jamovi.org/), a free open-source graphical user interface for R software. A p-value of $<0.05$ was considered statistically significant.

\section{RESULTS}

\section{Rate of completion of the program}

Twenty-five subjects (mean age, $67.3 \pm 12.5$ years) were included in the study. The types of disability were physical impairment $(n=5)$, auditory impairment $(n=12)$, brain impairment $(n=1)$, and visual impairment $(n=7)$. During the 12-week program, three exercise sessions were canceled, resulting in a final number of 21 exercise sessions. Seventeen (68\%) of the 25 subjects completed the program, attending $70 \%$ or more of the exercise sessions. For those who completed the exercise program, the number of absences in the exercise sessions ranged from 2 to 6 , leading to an attendance rate of $71 \%-90 \%$.

There was no significant difference in age or sex between the group that completed the program and the group that did not ( $\mathrm{p}=0.6$ and $\mathrm{p}=0.432$, respectively)
(Table 3). There was also no significant difference in the completion rate according to type of disability $(\mathrm{p}=0.384)$. One subject did not attend for the final assessment because of pain following a road traffic accident and was excluded from the analysis (Fig. 1). Finally, 16 subjects who completed the final assessment were included in further analysis.

Known cardiovascular disorders and cardiovascular risk factors in subjects who completed the final assessment

Among the 16 subjects who completed the final assessment, 8 had cardiovascular, renal, or metabolic disease (Table 4). Three subjects had a history of cardiovascular disease: 1 subject with a myocardial infarction, 1 subject

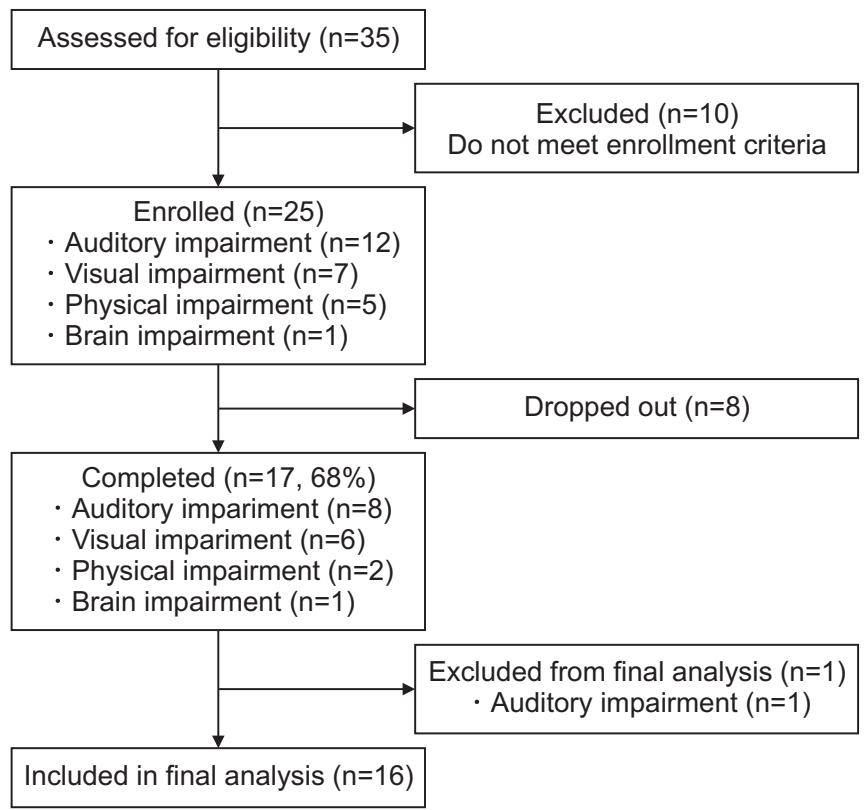

Fig. 1. Flow chart showing the enrollment process.

Table 3. Distributions of age, sex, and type of disability between subjects who completed the study and those who dropped out $(\mathrm{n}=25)$

\begin{tabular}{|c|c|c|c|c|}
\hline & All $(n=25)$ & Completed $(\mathrm{n}=17)$ & Dropped out $(n=8)$ & p-value \\
\hline Age (yr) & $67.3 \pm 12.5$ & $67.2 \pm 13.8$ & $67.6 \pm 10.0$ & $0.600^{\mathrm{a})}$ \\
\hline Sex, male & 16 & 10 & 6 & $0.432^{b)}$ \\
\hline \multicolumn{5}{|l|}{ Type of disability } \\
\hline Auditory impairment & 12 & 8 & 4 & $0.384^{\mathrm{c})}$ \\
\hline Visual impairment & 7 & 6 & 1 & \\
\hline Physical or brain impairment & 6 & 3 & 3 & \\
\hline
\end{tabular}

Values are presented as mean \pm standard deviation.

${ }^{\mathrm{a})}$ Mann-Whitney U test, ${ }^{\mathrm{b})} \chi^{2}$ test, ${ }^{\mathrm{c})}$ Fisher exact test. 
Sora Baek, et al.

Table 4. Baseline characteristics of subjects included in the final analysis $(n=16)$

\begin{tabular}{lcc}
\hline & All $(\mathbf{n}=\mathbf{1 6})$ & Visual or auditory impairments $(\mathbf{n}=\mathbf{1 3})$ \\
\hline Known cardiovascular, renal, or metabolic disorders & 8 & 6 \\
\hline Myocardial infarction & 1 & 1 \\
\hline Angina & 1 & 1 \\
\hline Stroke & 1 & 0 \\
\hline Renal disorder & 2 & 5 \\
\hline Diabetes mellitus & 6 & \\
Cardiovascular risk factors & & 10 \\
\hline Age & 13 & 1 \\
\hline Family history & 2 & 0 \\
\hline Cigarette smoking & 0 & 5 \\
\hline Sedentary lifestyle & 5 & 8 \\
\hline Obesity & 11 & 5 \\
\hline Hypertension & 7 & 5 \\
\hline Dyslipidemia & 5 & $6^{\text {b) }}$ \\
\hline Prediabetes and known diabetes mellitus & $8^{\text {a) }}$ & $2-5$ \\
\hline Number of risk factors (min-max) & $2-5$ & \\
\hline
\end{tabular}

${ }^{\text {a) }}$ Prediabetes $(\mathrm{n}=2)$ and known diabetes mellitus $(\mathrm{n}=6)$.

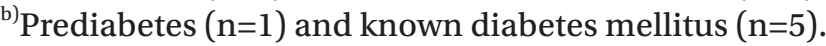

with an angina, and 1 subject with a stroke. Two subjects had renal disease and 6 had diabetes mellitus. Three subjects with a myocardial infarction, stroke, or renal disease also had diabetes mellitus; the final number of subjects with known disorders was 8 . Neither of the subjects with a known cardiovascular, renal, or metabolic disease had a sedentary lifestyle, and they were performing moderateintensity exercise more than three times per week.

Sixteen subjects who completed the final assessment had between 2 and 5 cardiovascular risk factors (Table 4 ). Age ( $\geq 45$ years for men, $\geq 55$ years for women) was the most frequent risk factor $(\mathrm{n}=13 ; 81.3 \%)$, followed by obesity $(\mathrm{n}=11 ; 68.8 \%)$. There were 8 subjects with diabetes mellitus and 2 patients with prediabetes (HbAlc of $5.7 \%-6.4 \%)$. None of the subjects smoked.

Six of the patients in the subgroup with visual or auditory impairment $(n=13)$ had cardiovascular, renal, or metabolic disease (Table 4). The number of cardiovascular risk factors in this subgroup ranged from 2 to 5 .

\section{Outcome measurements}

BMI and percent body fat were significantly decreased at the end of the exercise program in the 16 subjects who completed the final assessment $(\mathrm{p}<0.05)$. There was no significant change in waist circumference, resting blood pressure, or heart rate, or in fasting blood sugar, HbAlc, total cholesterol, or triglycerides. Physical activity (MET minutes per week) derived from the International Physical Activity Questionnaire was increased after the program; however, the change did not reach statistical significance $(p=0.065)$. The EQ-5D-3L index scores indicated no significant changes in quality of life. There was also no significant change in functional capacity assessed by the Korean Activity Scale/Index score. There was a significant increase in the number of 30 -second sit-tostand repetitions performed $(\mathrm{p}=0.034)$ but no significant change in 6MWD or handgrip strength (Table 5).

In the subgroup with visual or auditory impairment $(\mathrm{n}=13), \mathrm{BMI}$ and percent body fat were significantly decreased after the exercise program $(\mathrm{p}<0.05)$. The number of 30-second sit-to-stand repetitions increased in this group after the program; the increase was not statistically significant $(\mathrm{p}=0.135)$ (Table 5).

\section{DISCUSSION}

We have implemented a community-based aerobic and resistive exercise program for people with disabilities in Cheorwon-gun, where there is no cardiac rehabilitation center. In this study, 17 (68\%) of 25 subjects completed 
Table 5. Outcomes of subjects included in the final analysis $(n=16)$

\begin{tabular}{|c|c|c|c|c|c|c|}
\hline & \multicolumn{3}{|c|}{ All subjects $(n=16)$} & \multicolumn{3}{|c|}{ Visual or auditory impairments $(n=13)$} \\
\hline & Baseline & After intervention & p-value & Baseline & After intervention & p-value \\
\hline $\operatorname{BMI}\left(\mathrm{kg} / \mathrm{m}^{2}\right)$ & $26.4 \pm 3.6$ & $25.5 \pm 3.6$ & $<0.001$ & $26.4 \pm 4.0$ & $25.6 \pm 4.0$ & 0.005 \\
\hline Waist circumference $(\mathrm{cm})$ & $90.1 \pm 10.8$ & $91.5 \pm 11.9$ & 0.683 & $90.0 \pm 11.2$ & $91.1 \pm 13.2$ & 0.485 \\
\hline Percent body fat (\%) & $35.4 \pm 6.4$ & $34.2 \pm 5.9$ & 0.014 & $36.9 \pm 5.3$ & $35.4 \pm 5.5$ & 0.007 \\
\hline Muscle mass (kg) & $23.3 \pm 4.9$ & $23.1 \pm 4.7$ & 0.260 & $22.4 \pm 5.0$ & $22.3 \pm 4.9$ & 0.798 \\
\hline SBP (mmHg) & $131.1 \pm 17.9$ & $128.8 \pm 19.2$ & 0.431 & $128.5 \pm 18.1$ & $124.2 \pm 18.0$ & 0.234 \\
\hline DBP (mmHg) & $76.8 \pm 8.4$ & $75.8 \pm 10.3$ & 0.683 & $76.5 \pm 9.0$ & $73.4 \pm 9.0$ & 0.135 \\
\hline Heart rate (bpm) & $76.0 \pm 11.2$ & $73.4 \pm 7.9$ & 0.412 & $78.9 \pm 9.2$ & $73.7 \pm 8.2$ & 0.107 \\
\hline $\begin{array}{l}\text { Fasting blood sugar } \\
(\mathrm{mg} / \mathrm{dL})\end{array}$ & $96.9 \pm 25.4$ & $96.5 \pm 31.2$ & 0.884 & $97.8 \pm 28.4$ & $99.3 \pm 34.2$ & 0.753 \\
\hline $\operatorname{HbAlc}(\%)$ & $5.91 \pm 0.94$ & $6.03 \pm 0.99$ & 0.249 & $5.89 \pm 1.03$ & $6.01 \pm 1.06$ & 0.213 \\
\hline Total cholesterol (mg/dL) & $171.50 \pm 48.70$ & $174.88 \pm 41.60$ & 0.504 & $170.10 \pm 53.60$ & $169.50 \pm 44.60$ & 1.000 \\
\hline Triglycerides (mg/dL) & $106.9 \pm 53.8$ & $123.9 \pm 49.9$ & 0.106 & $109.5 \pm 58.9$ & $119.1 \pm 50.9$ & 0.184 \\
\hline EQ-5D-3L index & $0.835 \pm 0.206$ & $0.845 \pm 0.111$ & 0.795 & $0.907 \pm 0.065$ & $0.861 \pm 0.088$ & 0.058 \\
\hline MET-minute/week & $1,649.2 \pm 1,566.6$ & $2,812.9 \pm 2,279.4$ & 0.065 & $1,629.8 \pm 1,250.7$ & $2,748.4 \pm 2,232.2$ & 0.133 \\
\hline KASI score & $45.6 \pm 22.9$ & $43.2 \pm 15.8$ & 0.558 & $46.5 \pm 23.2$ & $41.7 \pm 14.3$ & 0.266 \\
\hline 6MWD (m) & $403.4 \pm 101.1$ & $417.4 \pm 133.0$ & 0.535 & $414.5 \pm 98.6$ & $417.5 \pm 139.1$ & 0.834 \\
\hline \multicolumn{7}{|l|}{ Handgrip strength (kgf) } \\
\hline Right & $22.8 \pm 13.3$ & $25.3 \pm 10.1$ & 0.369 & $21.7 \pm 14.3$ & $23.2 \pm 9.2$ & 0.635 \\
\hline Left & $20.5 \pm 11.3$ & $23.1 \pm 9.3$ & 0.382 & $20.0 \pm 12.0$ & $22.2 \pm 8.6$ & 0.610 \\
\hline $\begin{array}{l}\text { 30-second sit-to-stand test } \\
\text { (stands) }\end{array}$ & $11.8 \pm 5.0$ & $15.1 \pm 4.8$ & 0.034 & $12.2 \pm 5.4$ & $14.9 \pm 5.1$ & 0.135 \\
\hline
\end{tabular}

Values are presented as mean \pm standard deviation.

BMI, body mass index; SBP, systolic blood pressure; DBP, diastolic blood pressure; HbAlc, glycated hemoglobin; EQ5D-3L, European Quality of Life-5 Dimension-3-Level version; KASI, Korean Activity Scale/Index; 6MWD, 6-minute walk distance.

the 3-month program. After the end of the program, there were significant decreases in BMI and percent body fat and a significant increase in sit-to-stand strength. This is the first study to confirm the feasibility of a communitybased aerobic and resistive exercise program in persons with disabilities.

In a previous study, a 6-month strength training program improved the physical functional performance in people with physical disabilities [9]. The study subjects included older women with coronary heart disease who were assessed using the 36-Item Short Form (SF-36), and those with a physical function score of $<85$ were considered to have a physical disability [9]. The physical function domain of the SF-36 comprises 10 items encompassing physical difficulties [27], and the physical function score is reportedly associated with objective physical strength and walking performance [28]. In contrast, we recruited subjects with a disability registered under the Disability Welfare Act, which requires both a decreased functional status and medically confirmed impairments. To our knowledge, there is no report on the effects of cardiac rehabilitation in people with registered physical disabilities.

People with disabilities are at greater risk of health problems that are often preventable. Many reports indicate that health status is poorer in people with disabilities than in the general population. Moreover, compared with the general population, people with disabilities are significantly less likely to access health services; people with brain or physical impairment, in particular, have been shown to have more unmet healthcare needs than do the general population [13]. Such low use of health resources and low satisfaction rates are largely attributable to restrictions imposed by limitations in mobility [29]. 
One study found that people with disabilities were more likely than members of the general population to report difficulty in accessing healthcare services ( $14.8 \%$ vs. $4.2 \%$ ) [29]. The most frequently reported reasons for not accessing essential medical services were lack of money (58.8\%) and transport (18.6\%) [29].

Physical disability is associated with a decrease in physical activity levels. Furthermore, people with a physical disability are at increased cardiovascular risk [5] and are more likely to have risk factors for heart disease, such as obesity [3]. People with physical disabilities are also at high risk of developing heart disease [6,7]. Cardiac rehabilitation is well known to be effective for primary and secondary prevention of cardiovascular disease [8]. However, ongoing participation in cardiac rehabilitation is important, and distance from a cardiac rehabilitation center is an important barrier [30], particularly for people with physical disabilities. Community-based rehabilitation has been an important strategy in responding to the needs of people with disabilities.

The 6MWD test is a simple test that requires no specialized equipment or advanced training for the examiner. It assesses the submaximal functional capacity level of an individual and is a reliable test of functional exercise capacity in the healthy elderly [31] and people with a variety of disease states, including heart failure [32]. In this study, the measurement of 6MWD was modified from the original recommendations. Although the 6MWD is recommended to be tested on a hard floor, we tested it on a soft floor. This difference in environment presumably affected the reliability of the 6MWD test. In this study, there was no significant difference in the 6MWD before and after the program, but the test method may have influenced the results. Many buildings in the community, including health centers, do not have long corridors to implement the 6MWD. This study also used the indoor gym next door as an evaluation site due to these limitations. If an appropriate space for the 6MWD test is difficult to secure, a sit-to-stand test, which does not require much space, may be more useful in a community setting.

The present study recruited people with physical disabilities as subjects, and they exhibited a significant increase in the number of 30-second sit-to-stand repetitions. The 30 -second chair stand is a reliable and valid indicator of lower body strength in active, communitydwelling older adults [25]. The number of stands in 30 seconds has shown a moderate correlation with leg-press strength ( $\mathrm{r}=0.77$ ) [25]. Therefore, the 30 -second sit-tostand test, which measures the strength and endurance of the lower extremities, may be a useful outcome measurement in a person with a physical disability undergoing cardiac rehabilitation.

In this study, there were significant reductions in BMI and percent body fat after a 12-week exercise program. There was a tendency toward increased physical activity measured using the IPAQ after the program. On the other hand, other modifiable risk factors, including fasting blood glucose, HbAlc, total cholesterol, and triglycerides, were not significantly changed. Nutritional education was not included in this educational program, which may have influenced the results. The inclusion of nutritional education should be considered when implementing community cardiac rehabilitation education programs in the future.

This study has several limitations. First, we included subjects with physical disabilities, and not all the subjects had cardiovascular disorders. We aimed to assess the feasibility of a community-based aerobic and resistive exercise program conducted in a public health center for people with physical disabilities. In the future, a program for people with both physical disabilities and cardiovascular disorders needs to be studied. Second, we implemented a public health center-based program, where the cardiopulmonary exercise test was not available. Therefore, we only included the subjects who could start moderate intensity exercise without medical clearance according to the existing ACSM guidelines. If community-based cardiac rehabilitation is implemented in an institution, it should be performed after cardiopulmonary exercise testing. Third, although we aimed to show the effectiveness of public health center-based cardiac rehabilitation in subjects with physical disabilities, the subjects of this study had mild physical disabilities and were able to walk independently. Considering that a public health center is not a hospital with specialized cardiac rehabilitation facilities, we only included people with mild disabilities. However, people with more severe physical disabilities are considered to have more limited access to public health centers. The development of cardiac rehabilitation programs tailored for individuals with more severe disabilities is necessary in the future. Fourth, we performed the $6 \mathrm{MWD}$ test on a soft flat surface to lower the risk of 
falls. However, according to the recommended protocol, the 6MWD test is preferably performed on a flat corridor with a hard surface [26]. Thus, the floor condition may have affected our results. Fifth, the subjects exhibited diverse types of disabilities, including physical impairment, auditory impairment, brain impairment, and visual impairment. We suggest that a larger trial should include people with different types of disabilities separately.

In conclusion, people with visual, auditory, or physical/brain impairments who completed a 3-month community-based aerobic and resistive exercise program for primary prevention of cardiovascular disease achieved significant reductions in BMI and percent body fat and a significant increase in sit-to-stand strength. The strength and endurance of the lower extremities may be a useful outcome measure in this population.

\section{CONFLICT OF INTEREST}

No potential conflict of interest relevant to this article was reported.

\section{ACKNOWLEDGMENTS}

This work was supported by the National Research Foundation of Korea (NRF) grant funded by the Korean government (MSIT) (No. NRF-2017R1C1B5076976).

We acknowledge the collaboration of staff working at the Cheorwon Public Health Center.

\section{AUTHOR CONTRIBUTION}

Conceptualization: Baek S, Ha Y. Methodology: Baek S, Ha Y, Mok J, Lee H, Song W. Funding acquisition: Baek S. Writing - original draft: Baek S, Ha Y. Writing - review and editing: Baek S. Approval of final manuscript: all authors.

\section{REFERENCES}

1. Centers for Disease Prevention and Control. Disability and health overview [Internet]. Washington, DC: Centers for Disease Prevention and Control; c2020 [cited 2021 May 1]. Available from: https://www.cdc.gov/ ncbddd/disabilityandhealth/disability.html.

2. Achieve Australia. What is a physical disability? [In- ternet]. North Ryde, Australia: Achieve Australia; c2019 [cited 2021 May 1]. Available from: https:// achieveaustralia.org.au/ndis-overview-and-faqs/ physical-disability/.

3. Centers for Disease Prevention and Control. Disability and obesity [Internet]. Washington, DC: Centers for Disease Prevention and Control; c2019 [cited 2021 May 1]. Available from: https://www.cdc.gov/ ncbddd/disabilityandhealth/obesity.html.

4. American College of Sports Medicine. ACSM's guidelines for exercise testing and prescription. 10th ed. Philadelphia, PA: Wolters Kluwer; 2018.

5. Pinsky JL, Jette AM, Branch LG, Kannel WB, Feinleib M. The Framingham Disability Study: relationship of various coronary heart disease manifestations to disability in older persons living in the community. Am J Public Health 1990;80:1363-7.

6. Plichart M, Barberger-Gateau P, Tzourio C, Amouyel P, Peres K, Ritchie K, et al. Disability and incident coronary heart disease in older community-dwelling adults: the Three-City Study. J Am Geriatr Soc 2010;58:636-42.

7. Huang YY, Kung PT, Chiu LT, Tsai WC. Related factors and incidence risk of acute myocardial infarction among the people with disability: a national population-based study. Res Dev Disabil 2015;36C:366-75.

8. Anderson L, Oldridge N, Thompson DR, Zwisler AD, Rees K, Martin N, et al. Exercise-based cardiac rehabilitation for coronary heart disease: Cochrane systematic review and meta-analysis. J Am Coll Cardiol 2016;67:1-12.

9. Brochu M, Savage P, Lee M, Dee J, Cress ME, Poehlman ET, et al. Effects of resistance training on physical function in older disabled women with coronary heart disease. J Appl Physiol (1985) 2002;92:672-8.

10. Jeon B, Kwon S, Kim H. Health care utilization by people with disabilities: a longitudinal analysis of the Korea Welfare Panel Study (KoWePS). Disabil Health J 2015;8:353-62.

11. Im HW, Baek S, Jee S, Ahn JM, Park MW, Kim WS. Barriers to outpatient hospital-based cardiac rehabilitation in Korean patients with acute coronary syndrome. Ann Rehabil Med 2018;42:154-65.

12. Kim SH, Byun YC, Sohn CK, Lee YH, Lee MK, Lee SH, et al. The 2011 National Survey of the Disabled Persons. Sejong, Korea: Korea Institute for Health and 
Social Affairs; 2012.

13. Hwang B, Chun SM, Park JH, Shin HI. Unmet healthcare needs in people with disabilities: comparison with the general population in Korea. Ann Rehabil Med 2011;35:627-35.

14. Ministry of Health and Welfare. Health and welfare statistical yearbook 2019. Sejong, Korea: Minister of Health and Welfare; 2019.

15. Baek S, Ha Y, Mok J, Park HW, Son HR, Jin MS. Community-based cardiac rehabilitation conducted in a public health center in South Korea: a preliminary study. Ann Rehabil Med 2020;44:481-92.

16. American College of Sports Medicine. ACSM's guidelines for exercise testing and prescription. 9th ed. Baltimore, MD: Lippincott Williams \& Wilkins; 2013.

17. Holden MK, Gill KM, Magliozzi MR. Gait assessment for neurologically impaired patients: standards for outcome assessment. Phys Ther 1986;66:1530-9.

18. Balady GJ, Williams MA, Ades PA, Bittner V, Comoss P, Foody JM, et al. Core components of cardiac rehabilitation/secondary prevention programs: 2007 update: a scientific statement from the American Heart Association Exercise, Cardiac Rehabilitation, and Prevention Committee, the Council on Clinical Cardiology; the Councils on Cardiovascular Nursing, Epidemiology and Prevention, and Nutrition, Physical Activity, and Metabolism; and the American Association of Cardiovascular and Pulmonary Rehabilitation. Circulation 2007;115:2675-82.

19. Pinkstaff S, Peberdy MA, Kontos MC, Finucane S, Arena R. Quantifying exertion level during exercise stress testing using percentage of age-predicted maximal heart rate, rate pressure product, and perceived exertion. Mayo Clin Proc 2010;85:1095-100.

20. TheraBand resistance band \& tubing instruction manual [Internet]. Akton, OH: Performance Health; 2012 [cited 2021 May 1]. Available from: http://www. thera-bandacademy.com/resource/x-showResource. aspx?id=1461.

21. TheraBand CLX consecutive loops [Internet]. Akton, OH: Performance Health; 2015 [cited 2021 May 1]. Available from: https://www.artzt.eu/media/ pdf/18/75/8f/Exercise-Instruction_Thera-Band-CLX_ English.pdf.
22. Sung J, On YK, Kim HS, Chae IH, Sohn DW, Oh BH, et al. Development of Korean activity scale/index (KASI). Korean Circ J 2000;30:1004-9.

23. Oh JY, Yang YJ, Kim BS, Kang JH. Validity and reliability of Korean version of International Physical Activity Questionnaire (IPAQ) short form. J Korean Acad Fam Med 2007;28:532-41.

24. Lee YK, Nam HS, Chuang LH, Kim KY, Yang HK, Kwon IS, et al. South Korean time trade-off values for EQ-5D health states: modeling with observed values for 101 health states. Value Health 2009;12:1187-93.

25. Jones CJ, Rikli RE, Beam WC. A 30-s chair-stand test as a measure of lower body strength in communityresiding older adults. Res Q Exerc Sport 1999;70:113-9.

26. ATS Committee on Proficiency Standards for Clinical Pulmonary Function Laboratories. ATS statement: guidelines for the six-minute walk test. Am J Respir Crit Care Med 2002;166:111-7.

27. Ware JE, Snow KK, Kosinski M, Gandek B. SF-36 health survey: manual and interpretation guide. Boston, MA: The Health Institute, New England Medical Center; 1993.

28. Syddall HE, Martin HJ, Harwood RH, Cooper C, Aihie Sayer A. The SF-36: a simple, effective measure of mobility-disability for epidemiological studies. J Nutr Health Aging 2009;13:57-62.

29. Lee JE, Kim HR, Shin HI. Accessibility of medical services for persons with disabilities: comparison with the general population in Korea. Disabil Rehabil 2014;36:1728-34.

30. Bakhshayeh S, Sarbaz M, Kimiafar K, Vakilian F, Esla$\mathrm{mi}$ S. Barriers to participation in center-based cardiac rehabilitation programs and patients' attitude toward home-based cardiac rehabilitation programs. Physiother Theory Pract 2021;37:158-68.

31. Troosters T, Gosselink R, Decramer M. Six minute walking distance in healthy elderly subjects. Eur Respir J 1999;14:270-4.

32. Faggiano P, D'Aloia A, Gualeni A, Lavatelli A, Giordano A. Assessment of oxygen uptake during the 6 -minute walking test in patients with heart failure: preliminary experience with a portable device. Am Heart J 1997;134(2 Pt 1):203-6. 